\title{
Uncertainties in nebular helium abundances
}

\author{
Evan D. Skillman ${ }^{1}$ \\ ${ }^{1}$ Astronomy Department, University of Minnesota, \\ 116 Church St. SE, Minneapolis, MN, USA \\ email: skillman@astro.umn.edu
}

\begin{abstract}
Efforts to determine the primordial helium abundance via observations of metal poor HII regions have been limited by significant uncertainties. Because of a degeneracy between the solutions for density and temperature, the precision of the helium abundance determinations is limited. Spectra from the literature are used to show the effects of new atomic data and to demonstrate the challenges of determining precise He abundances. Several suggestions are made for meeting these challenges.
\end{abstract}

Keywords. nucleosynthesis, abundances, cosmological parameters, early universe, ISM: HII regions

\section{Introduction: In defense of Olive \& Skillman (2004)}

Fig. 1 shows the historical evolution of the observational determinations of the primordial helium abundance compared to the present day value inferred from WMAP observations and BBN calculations. One well known effect shown in the plot is the small error bars relative to the differences between determinations and relative to the currently favored value. In 2004, Keith Olive and I (Olive \& Skillman 2004, hereafter OS04) attempted to account for most of the important statistical and systematic uncertainties, and, in a reanalysis of data from the literature (Izotov \& Thuan 1998; Peimbert, Peimbert, \& Ruiz 2000), found significantly larger uncertainties for helium abundance determinations for individual objects and a resulting larger uncertainty in the primordial helium abundance. In the plot, this result appears as a giant step backwards as the uncertainty in the primordial helium abundance is comparable to uncertainties associated with determinations from the 1980's. Part of the large uncertainty is due to concentrating on a small sample of "high quality" spectra of the lowest metallicity objects. This was to avoid two statistical effects which we felt were unsound. Including a large number of objects will reduce the error in the primordial helium abundance, even if some of those objects have relatively lower quality spectra. Additionally, including objects at higher metallicity will reduce the error on the primordial helium abundance because the extrapolation to zero metallicity will have a smaller error. However, this increases the dependence on the assumption of a linear relationship between the helium abundance and the oxygen (or nitrogen) abundance. By avoiding these two effects, we felt that the derived uncertainty in the primordial helium abundance better characterized the true uncertainty. Notably, a significant factor in the increased uncertainty of OS04 was simply larger uncertainties on the helium abundances of individual objects due to including neglected terms and running Monte Carlo analyses to account for degeneracies in minimization solutions.

An important result of the Monte Carlo analysis was the discovery of degeneracies in the sense that the physical conditions in the HII regions were not always independently constrained (Olive \& Skillman 2001; Olive \& Skillman 2004). In particular, the temperature and density exhibited a trade-off whereby increasing the temperature while decreasing the density would leave the $\chi^{2}$ relatively unchanged. The main reason for this 


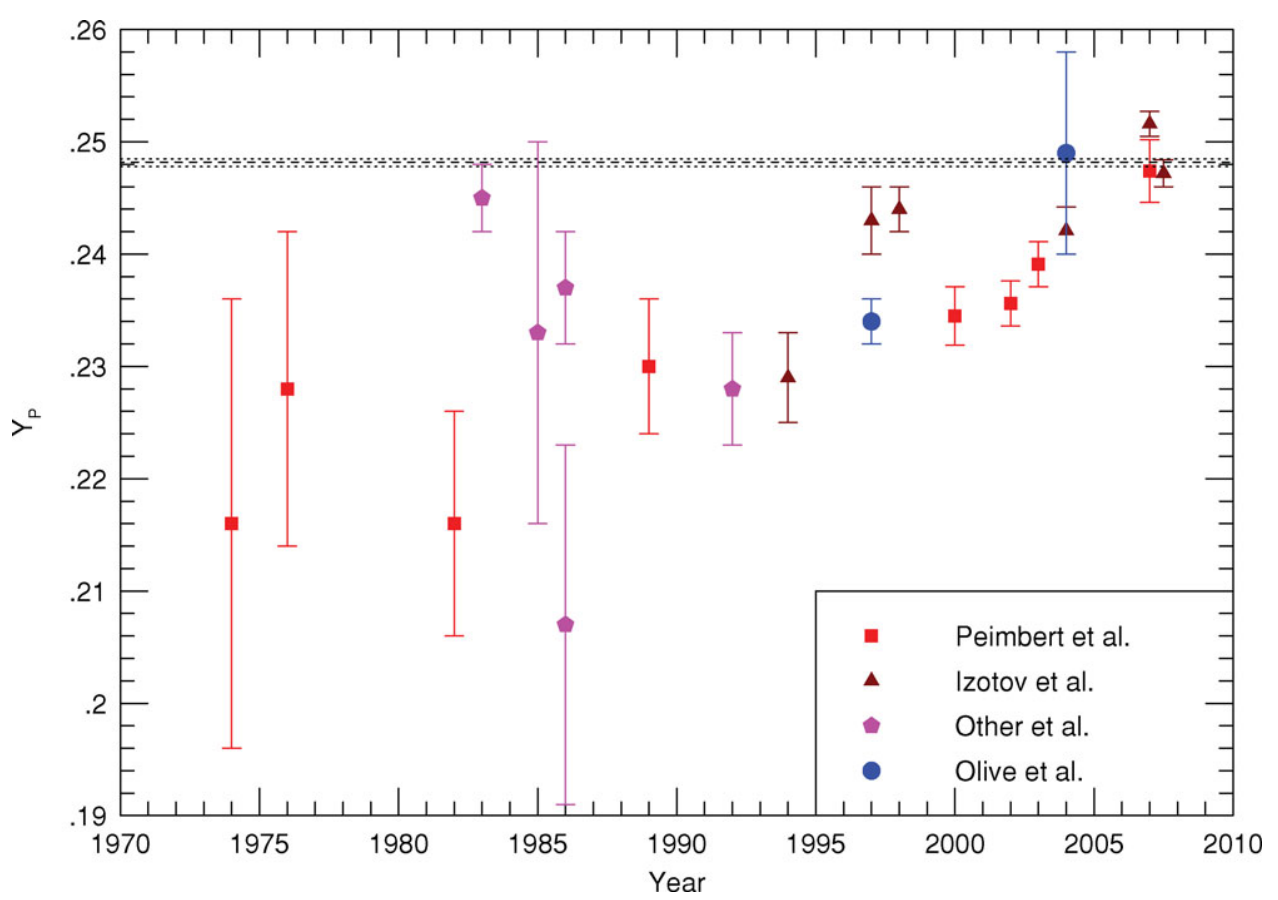

Figure 1. Historical evolution of the observational determinations of the primordial helium abundance. The solid horizontal line with dashes shows the value inferred from WMAP observations and $\mathrm{BBN}$ calculations.

is that four of the HeI emission lines used to derive the helium abundance show similar exponential dependencies on temperature and density.

We demonstrate the importance of the degeneracy between temperature and density in Figure 2. Here, we have generated a synthetic spectrum using typical physical conditions and a helium abundance. Values of the helium abundance and a corresponding $\chi^{2}$ were then derived from this spectrum keeping all parameters fixed, but varying the density and temperature. The impact of trading the density against the temperature can be clearly seen in Figure 2 which illustrates a well constrained quotient of density and temperature but a $\triangle \chi^{2}=2.3$ boundary spanning a temperature range of $5500 \mathrm{~K}$, a density range of $275 \mathrm{~cm}^{-3}$, and an abundance variation of $10 \%$. The shallow, extended minimum results in a large range of best fit parameters for the density and temperature upon the Monte Carlo perturbation, and the abundance gains a much larger uncertainty (see Figure 3). Only by solving for these two parameters simultaneously can this degeneracy be discovered.

\section{New advances in helium abundance analysis}

With University of Minnesota graduate student Erik Aver, Keith Olive and I have investigated improvements to the code that we used in 2004. This was, in part, in response to recent improvements to the atomic data (Porter, Ferland, \& MacAdam 2007) which have resulted in significant changes to the derived helium abundances of HII regions (Izotov et al. 2007; Peimbert, Luridiana, \& Peimbert 2007). Additionally, we have investigated combining the minimizations, previously based on the hydrogen lines and the helium lines separately, into a single minimization, which is inherently self-consistent. The single minimization allows us to investigate the correction for the collisional emission of a small population of neutral hydrogen (e.g., Davidson \& Kinman 1985; Skillman \& 


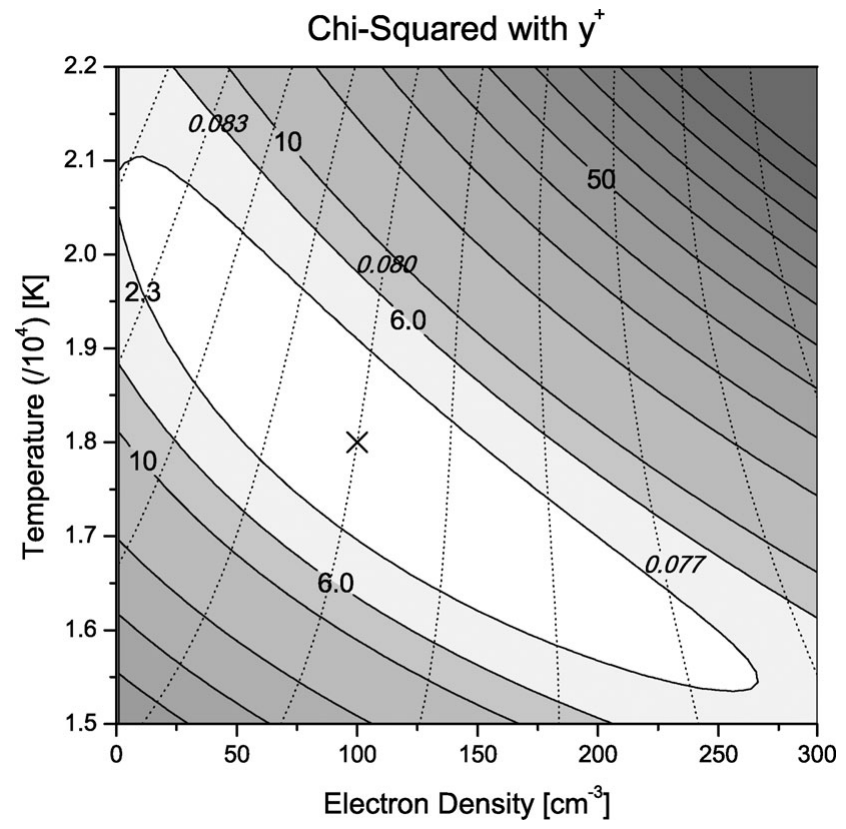

Figure 2. A plot of the derived helium abundance as a function of the temperature and density for a synthetically generated spectrum. Impressed on this diagram is a contour plot of $\chi^{2}$ versus density and temperature for the same spectrum. The extension of the $\chi^{2}=2.3$ contour with a strong negative correlation highlights the degeneracy between density and temperature. That the $\chi^{2}$ and abundance contours are nearly perpendicular demonstrates the impact of the degeneracy on the abundance determination $( \pm 5 \%)$. From Aver, Olive, \& Skillman (2010).

Kennicutt 1993; Stasińska \& Izotov 2001; Luridiana et al. 2003). Finally, the wavelength dependence in underlying stellar absorption can be included (initial previous work assumed identical values of equivalent width of underlying absorption for all $\mathrm{H} \mathrm{I}$ or He I emission lines, regardless of wavelength) as has been investigated previously by Izotov et al. (2007). Detailing the incorporation of these four effects and investigation of their impact is the primary aim of a forthcoming paper (Aver, Olive, \& Skillman 2010), and here I will provide a summary of the main results.

The updated emissivities, including the collisional corrections, in general raise the calculated abundance of helium (in agreement with Izotov et al. 2007; Peimbert, Luridiana, \& Peimbert 2007). However, because the changes in different lines are qualitatively different, the update does not categorically raise the abundance. For $\lambda 4471,5876$, and 6678 , the $\mathrm{H} \beta$ to He emissivity is increased rather uniformly (2-3\%) across the relevant temperature range, and all three lines exhibit a gradual increase with temperature. The behavior of $\lambda 4026$ is similar except that the new emissivity is shifted to lower values. $\lambda 3889$ is more complicated in that the emissivity is reduced at low temperatures but is enhanced at $T \geqslant 16,000 \mathrm{~K} . \lambda 7065$ is similar though the enhancement begins at 13,000 K. Broadly though, both $\lambda 3889$ and $\lambda 7065$ as well as $\lambda 4471$ track the BSS emissivities more closely (within $\sim 2 \%$ ) than the other three lines. The three strongest lines with the smallest relative errors will produce a larger abundance, thus raising the average abundance. However, the changes to $\lambda \lambda 3889,7065$, and 4471 affect the derived physical conditions in the nebula, and, in some cases, drive the derived abundance to lower values, opposite to the trend for the three strongest lines. 


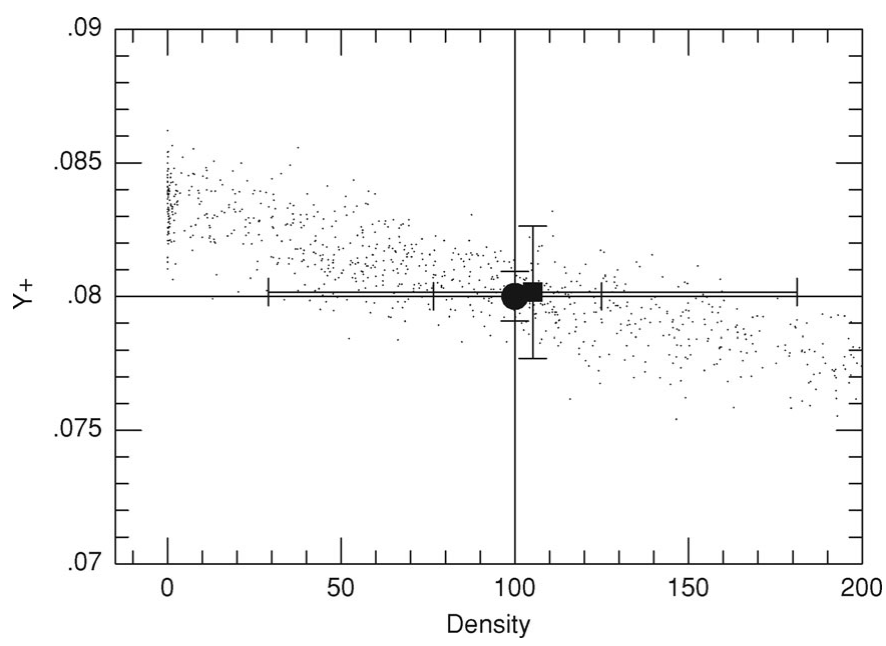

Figure 3. Plot of 1000 Monte Carlo solutions based upon a synthetic spectrum. The solid circle with error bars marks the original (direct) solution; while the solid square with error bars marks the average of the Monte Carlo solutions. Upon performing the Monte Carlo, the large range in density solutions (and correspondingly temperature solutions), gives a much larger density uncertainty, resulting in a marked increase in the abundance uncertainty (from 1\% to $3 \%$ ). From Olive, \& Skillman (2001).

The changes to the atomic data for helium emissivities were much larger than ever accounted for in previous work. In most cases these uncertainties were regarded as insignificant when compared with the other uncertainties. Benjamin, Skillman, \& Smits (1999) estimated a minimum uncertainty of $1.5 \%$ and this value is comparable to the changes due to the use of the new emissivities. This change alone validates the main conclusion of Olive \& Skillman (2004) that the uncertainties in the derived helium abundances of individual objects were underestimated.

The combining of both the helium and hydrogen lines into a single minimization program has only small impact as long as collisional excitation of neutral hydrogen is not included. This is because the relative fluxes of the hydrogen emission from recombination has very low dependencies on density and temperature, so the hydrogen lines do not play into the density/temperature degeneracy. However, once collisional excitation of neutral hydrogen is included, then the strengths of the hydrogen lines gain a new temperature dependence, and it is crucial to include them in the minimization. A three way degeneracy between electron density, temperature, and the neutral hydrogen fraction emerges. These degeneracies are shown in Figure 4 which shows the Monte Carlo results for NGC 346. Plotted are the individual results for density, temperature, and neutral hydrogen fraction for $1000 \mathrm{MC}$ realizations. As one can see, the solutions map out a wide range for these parameter values. The neutral hydrogen fraction can be increased to compensate for a decrease in the collisional to recombination rate which is itself only temperature dependent. Furthermore, the neutral hydrogen collisional emission correction can have a surprisingly significant effect in raising the abundance. Since the collisional correction for $\mathrm{H} \beta$ multiplies each helium line abundance, it multiplicatively raises the abundance but does not change the value of the corresponding $\chi^{2}$. As a result, the helium abundances of all the objects that we studied increased.

The uncertainty in the choice of correction for underlying absorption is chiefly mitigated by selecting only spectra with very high emission line equivalent widths. The main problems regarding underlying absorption arise when corrections are not included at all 


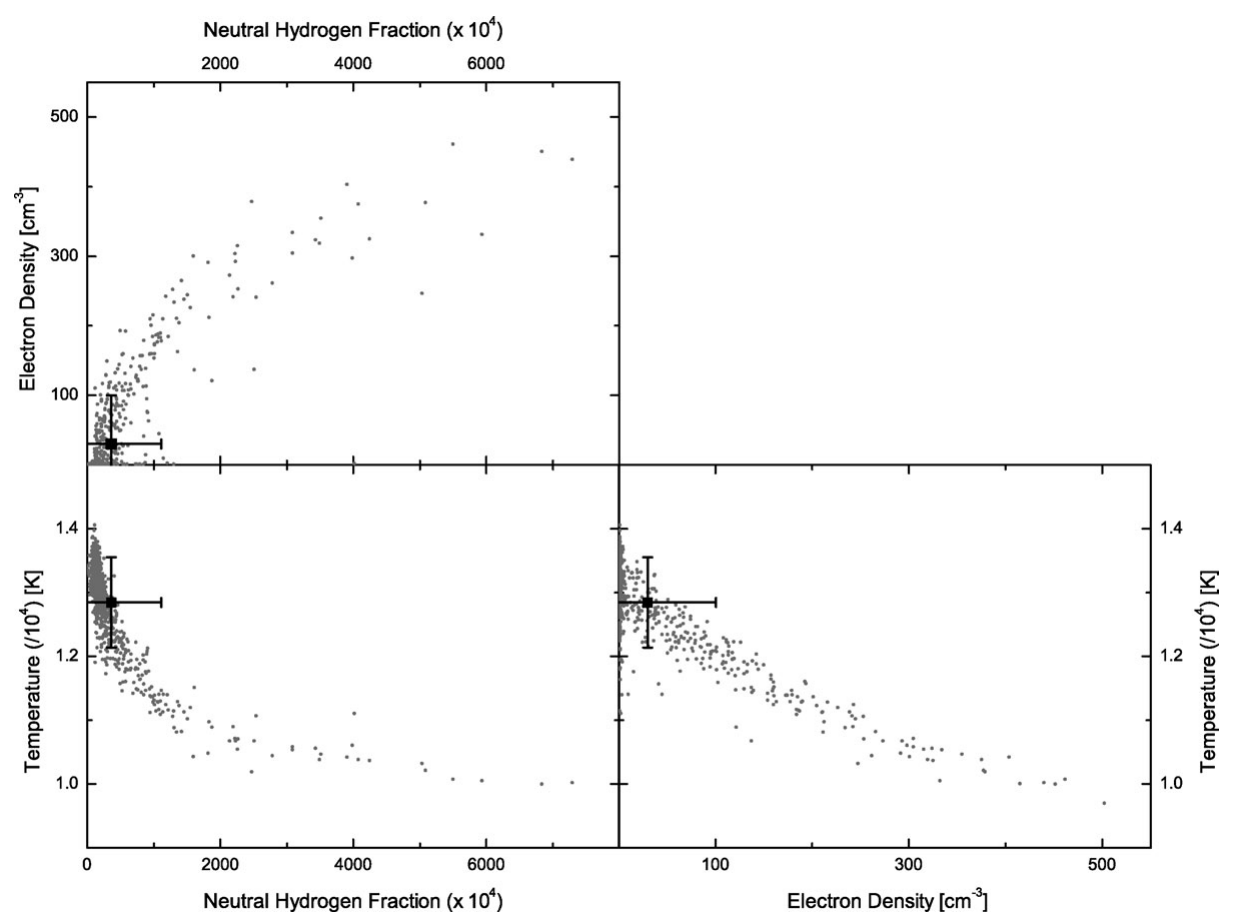

Figure 4. Plots of Monte Carlo results (1000 points) of density, temperature, and neutral hydrogen fraction for NGC 346. The strong correlation between the three parameters demonstrates the difficulty in solving for them independently. From Aver, Olive, \& Skillman (2010).

(cf. Skillman, Terlevich, \& Terlevich 1998 ). Even in the case of NGC 346, where a filament with no obvious exciting stars was observed (Peimbert, Peimbert, \& Ruiz 2000), Olive \& Skillman (2004) found evidence for significant underlying absorption. This was confirmed by Peimbert, Luridiana, \& Peimbert (2007). Once again, this underlines the importance of obtaining high quality spectra of the most promising targets.

Extending the previous work to allow for self-consistent analysis reflecting the common temperature and reddening parameters between the hydrogen and helium lines does not diminish the importance of the systematic uncertainties or the case for the larger errors determined via Monte Carlo. In fact, the addition of the neutral hydrogen collisional emission, introduced with a new physical parameter, the neutral hydrogen fraction, highlights that abundance errors may be even larger than previously thought. In addition to the need for observations to constrain systematic uncertainty as much as possible, we have found that relatively small systematic errors in the observed fluxes, even of the weakest lines, can have a significant impact on the derived value of $\mathrm{y}^{+}$. Clearly the highest quality spectra are critical for a meaningful analysis.

Ultimately, the sensitivity of the determination of the primordial helium abundance demands that analysis be self-consistent and comprehensive; unfortunately efforts in providing such an analysis yield calculated uncertainties that limit the utility of that determination. Nevertheless, fully understanding the terrain of potential obstacles is necessary to direct further measurement efforts so as to provide a reliable calculation of the primordial helium abundance. Apart from improving the quality of the spectra, the neutral hydrogen collisional correction for the weaker hydrogen lines can be easily improved from extended principal quantum number collisional strengths. Furthermore the use of an additional hydrogen line would benefit the neutral hydrogen fraction and 
hydrogen absorption since those two parameters are determined exclusively through the three hydrogen lines. The next Balmer line, H7 ( $\lambda 3971)$ is blended with Ne III $(\lambda 3967)$, and H8, blended with HeI $\lambda 3889$, would not be independent for analysis. This leaves the very weak $\mathrm{H} 9$ and $\mathrm{H} 10$ as the best candidates. The use of other emission lines may also be useful in lifting the error increasing degeneracy between density and temperature. However, this requires accurate metal line fluxes whose ratio is sensitive to the electron density or temperature in the applicable range. Most candidates are impaired by the weakness of their emission lines and ionization populations that are not characteristic of the majority of the HII region.

\section{References}

Benjamin, R. A., Skillman, E. D., \& Smits, D. P. 1999, ApJ, 514, 307

Davidson, K. \& Kinman, T. D. 1985, ApJS, 58, 321

Izotov, Y. I. \& Thuan, T. X. 1998, ApJ, 500, 188

Izotov, Y. I., Thuan, T. X., \& Stasinska, G. 2007, ApJ, 662, 15 (ITS07)

Luridiana, V., Peimbert, A., Peimbert, M., \& Cerviño, M. 2003, ApJ, 592, 846

Olive, K. A. \& Skillman, E. D. 2001, New Astronomy, 6, 119

Olive, K. A. \& Skillman, E. D. 2004, ApJ, 617, 29

Peimbert, M., Luridiana, V., \& Peimbert, A. 2007, ApJ, 666, 636 (PLP07)

Peimbert, M., Peimbert, A., \& Ruiz, M. T. 2000, ApJ, 541, 688

Porter, R. L., Ferland, G. J., \& MacAdam, K. B. 2007, ApJ, 657, 327 (PFM)

Skillman, E. D. \& Kennicutt, R. C. 1993, ApJ, 411, 655

Skillman, E. D., Terlevich, E., \& Terlevich, R. 1998, Spa. Sci. Rev., 84, 105

Stasińska, G. \& Izotov, Y. I. 2001, A\& $A$, 378, 817 